\title{
High Frequency Guided Wave Propagation in Monocrystalline Silicon Wafers
}

\author{
Marco Pizzolato a), Bernard Masserey ${ }^{\text {a) }}$, Jean-Luc Robyr ${ }^{\text {a) }}$ and Paul Fromme ${ }^{\text {b) }}$ \\ a) Department of Mechanical Engineering, University of Applied Sciences, Fribourg, Switzerland \\ ${ }^{b)}$ Department of Mechanical Engineering, University College London, UK
}

\begin{abstract}
Monocrystalline silicon wafers are widely used in the photovoltaic industry for solar panels with high conversion efficiency. The cutting process can introduce micro-cracks in the thin wafers and lead to varying thickness. High frequency guided ultrasonic waves are considered for the structural monitoring of the wafers. The anisotropy of the monocrystalline silicon leads to variations of the wave characteristics, depending on the propagation direction relative to the crystal orientation. Full three-dimensional Finite Element simulations of the guided wave propagation were conducted to visualize and quantify these effects for a line source. The phase velocity (slowness) and skew angle of the two fundamental Lamb wave modes (first anti-symmetric mode $\mathrm{A}_{0}$ and first symmetric mode $\mathrm{S}_{0}$ ) for varying propagation directions relative to the crystal orientation were measured experimentally. Selective mode excitation was achieved using a contact piezoelectric transducer with a custom-made wedge and holder to achieve a controlled contact pressure. The out-of-plane component of the guided wave propagation was measured using a noncontact laser interferometer. Good agreement was found with the simulation results and theoretical predictions based on nominal material properties of the silicon wafer.
\end{abstract}

Keywords: Silicon, Elastic Anisotropy, Lamb Wave Modes

\section{INTRODUCTION}

Solar photovoltaic electricity generation has become an important alternative to traditional fossil fuel resources. The further development of this renewable energy resource depends on higher conversion efficiency and lower production costs. Both of these aspects rely on the production of thinner silicon wafers that lower the solar panel costs and increase their electricity productivity. The thin silicon wafers used in the photovoltaic industry are very fragile. The manufacturing processes, from raw silicon crystal sawing up to the final assembly in solar panels, can potentially induce physical defects in the crystal such as scratches and cracks, and thus increase the probability of wafer breakage. In practice, the minimum wafer thickness in photovoltaics is determined by the breakage rates [1]. In this context, in-line detection of defects during the manufacturing process is crucial to prevent breakage during later stages of processing and therefore costly material losses (silicon wafers contribute up to $75 \%$ of the overall cost of solar cells). Several nondestructive methods exist to investigate cracks and structural defects in silicon crystals. These methods differ by their physical principle of detection: optical transmission and interferometry, thermography, impact testing, ultrasonic wave propagation, electro- and photo-luminescence imaging (for a review of techniques see $[2,3]$ ). However, only some of these methods are fast and sensitive enough to be used for in-line inspection purpose.

In this context, guided ultrasonic waves [4] have been proposed for the monitoring of silicon wafers during the production process. Guided waves can propagate over long distances compared to the thickness of the structure and have been used successfully in a structural health monitoring approach to detect and localize defects in plate structures [5, 6]. High frequency guided waves were employed to detect hidden damage [7] and fatigue cracks [8-10]. Crack detection in $525 \mu \mathrm{m}$ thick silicon wafers was achieved using non-contact laser excitation and measurement of the fundamental Lamb wave modes $\left(\mathrm{A}_{0}\right.$ and $\left.\mathrm{S}_{0}\right)$ at frequencies up to $2 \mathrm{MHz}$ [11]. Mono-crystalline and poly-crystalline silicon wafers $(200 \mu \mathrm{m}$ thickness) for solar cells were tested using non-contact air-coupled transducers at $200 \mathrm{kHz}$ excitation frequency. The measured amplitude drop of the Lamb waves in a B-scan configuration was used to detect cracks [12].

Health Monitoring of Structural and Biological Systems 2017, edited by Tribikram Kundu, Proc. of SPIE Vol. 10170, 101702B · (C) 2017 SPIE · CCC code: 0277-786X/17/\$18 · doi: 10.1117/12.2260035 
The anisotropic material properties of silicon lead to a dependency of the ultrasonic wave propagation characteristics on the direction relative to the crystal orientation. Theoretical calculations predict the energy focusing of longitudinal and transverse ultrasonic waves in anisotropic materials, including silicon [13]. The velocity dependency of bulk ultrasonic waves in thick silicon plates was measured experimentally, compared to theory [14], and inverted to obtain the anisotropic material properties [15]. The directional dependency of the zero group velocity of guided waves on $525 \mu \mathrm{m}$ thick silicon wafers was measured using a line laser source up to about $18 \mathrm{MHz}$ excitation frequency [16]. Results showed that the cut-off frequency of the $S_{1}$ mode varies with direction and that stronger amplitude was observed in directions with higher stiffness. Guided wave propagation in composite plates and $625 \mu \mathrm{m}$ thick silicon wafers was excited using a broadband laser source and measured using a shear piezoelectric transducer to study the variation in arrival time and amplitude with propagation direction for the $\mathrm{S}_{0}$ and $\mathrm{SH}$ wave modes [17]. Similarities exist to the guided wave propagation in composite plates, where more recently the concentration of energy along the stiffer fiber directions was analyzed [18] and the focusing of different modes was predicted [19].

In this contribution, the wave propagation of the fundamental guided wave modes $\left(\mathrm{A}_{0}\right.$ and $\left.\mathrm{S}_{0}\right)$ in thin monocrystalline silicon wafers was investigated at an excitation frequency of $5 \mathrm{MHz}$. The focus was on the angular dependence of the wave propagation characteristics, where the variation in velocity leads to the skewing of the wave propagation dependent on the orientation of the excitation to the crystal axes. Finite Element (FE) simulations were performed to predict the phenomena and compared to experimental results.

\section{BACKGROUND AND THEORY}

Theoretical calculation of the phase velocity of the fundamental Lamb modes in function of the angular orientation of the silicon crystal was performed using Disperse [20] for a $380 \mu \mathrm{m}$ thick silicon wafer characterized by three cubic symmetric stiffness constants: $\mathrm{C}_{11}=165.70 \mathrm{GPa}, \mathrm{C}_{12}=63.90 \mathrm{GPa}$ and $\mathrm{C}_{44}=79.56 \mathrm{GPa}$. The constants are determined for $<100>$ crystal orientation, corresponding to an azimuth angle $\varphi=0^{\circ}$. Figure 1a shows the dispersion diagram (phase velocity) for the two fundamental Lamb modes $\left(\mathrm{A}_{0}\right.$ and $\left.\mathrm{S}_{0}\right)$ for the $<100>$ and $<110>$ crystal orientations. The $<100>$ orientation (azimuth angle $\varphi=0^{\circ}$ ) corresponds to the lowest stiffness direction and thus slower velocities compared to the $<110>$ orientation (azimuth angle $\varphi=45^{\circ}$ ) with the highest stiffness. The variation in function of the crystallographic orientation is shown in Fig. $1 \mathrm{~b}$ as the phase slowness, the inverse of the phase velocity. Smaller variation of the $\mathrm{A}_{0}$ mode compared to the $\mathrm{S}_{0}$ mode can be observed, as well as the $45^{\circ}$ symmetry of the silicon crystal material properties. The skew angle (perpendicular to phase slowness curve) can be calculated and indicates the divergence of the wave propagation direction to the launching direction [21]. The variation of the phase velocity with wafer thickness is indicated in Fig. 1a by the 3 vertical lines, demonstrating that in principle accurate measurements of the phase velocity would allow a determination of the wafer thickness.

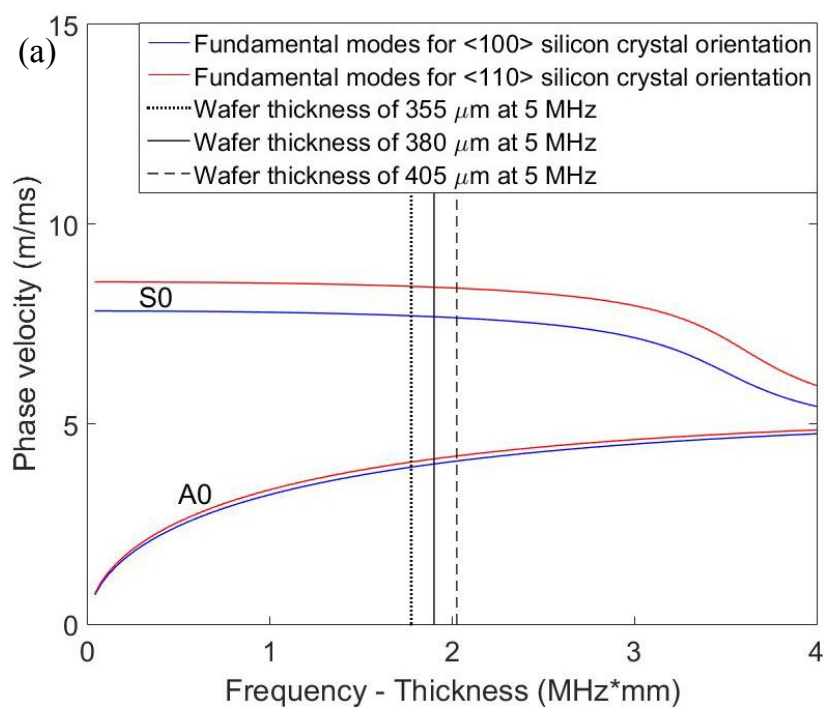

(b)

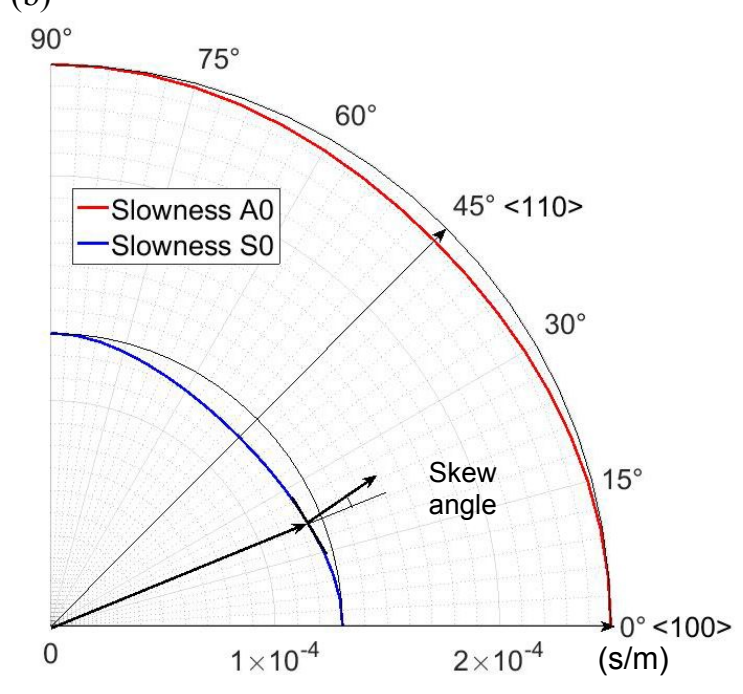

Fig. 1: (a) Phase velocity dispersion diagram for variation of wafer thickness and crystal orientation; (b) phase slowness for $\mathrm{A}_{0}$ and $\mathrm{S}_{0}$ modes at $5 \mathrm{MHz}$, wafer thickness of $380 \mu \mathrm{m}$, showing skew angle. 


\section{FINITE ELEMENT SIMULATIONS}

The propagation of the guided wave modes in the silicon wafers was simulated using three-dimensional (3D) FE models with linear brick elements $(\Delta \mathrm{x}=\Delta \mathrm{y}=50 \mu \mathrm{m}, \Delta \mathrm{z}=47.5 \mu \mathrm{m})$ in ABAQUS Explicit. The model of a $380 \mu \mathrm{m}$ thick silicon plate (size: $60 \mathrm{~mm}$ x $60 \mathrm{~mm}$ ) was implemented. Explicit time integration was used, and the element size and time step were chosen to adhere to the usual stability criteria. Line excitation of respectively the $\mathrm{A}_{0}$ and $\mathrm{S}_{0}$ Lamb wave modes was introduced at one edge of the plate. Approximating the transducer width of $5 \mathrm{~mm}$ used for the experiments, all nodes over a width of $5 \mathrm{~mm}$ at the center of the plate thickness were excited either using in-plane $\left(\mathrm{S}_{0}\right.$ mode $)$ or out-of-plane $\left(\mathrm{A}_{0}\right.$ mode) motion. The amplitude over the $5 \mathrm{~mm}$ excitation width was set to vary from 0.5 at the sides to 1 at the center using a Hanning distribution. The excitation pulse was set as a 12 cycle toneburst with a center frequency $f$ of $5 \mathrm{MHz}$, giving a wavelength $\lambda$ for the $A_{0}$ mode of about $0.8 \mathrm{~mm}$ and for the $S_{0}$ mode of approximately $1.6 \mathrm{~mm}$. The amplitude for both modes was monitored as the out-of-plane displacement at the top surface nodes on points in a rectangular area of $40 \mathrm{~mm}$ by $50 \mathrm{~mm}$ in front of the excitation to capture the propagation and wave skew of the guided wave mode pulses. For the phase velocity evaluation, the wave propagation was monitored on a line in front of the middle of the excitation. The time trace at each monitoring node was time gated to remove reflections from the plate edges. Fast Fourier Transform (FFT) was used to extract the complex magnitude (amplitude and phase information) at the center frequency of $5 \mathrm{MHz}$ for each monitoring node. Alternatively, the Hilbert transform was used to obtain the maximum of the envelope of the time trace at each monitoring node. The orthotropic (anisotropic) material properties were specified using the nominal material properties from literature stated above. For simulations of different excitation orientations relative to the crystallographic axes, the material properties were specified with a rotated axis system in ABAQUS, keeping the geometry the same.

\section{EXPERIMENTS}

Single crystal silicon wafers with $<100>$ crystallographic orientation were used for the measurements. The P-type silicon specimens are boron doped and have a diameter of $100 \mathrm{~mm}$ and nominal thickness of $380 \mu \mathrm{m}$. The guided waves were generated using an angle beam transducer, made of a commercial single element transducer and custom-made Nylon wedges with respectively $41^{\circ}\left(\mathrm{A}_{0}\right.$ mode) and $19^{\circ}$ angles $\left(\mathrm{S}_{0}\right.$ mode). The wedge angles were specially adapted to selectively excite the first fundamental Lamb modes $\left(\mathrm{A}_{0}\right.$ or $\left.\mathrm{S}_{0}\right)$. A holder was developed and used to secure the position, orientation and contact force of the angle beam transducer. A second holder guaranteed the orientation and position of the silicon wafer allowing $0.5^{\circ}$ angular orientation steps (Fig. 2). The excitation signal was defined as a sinusoidal pulse with $5 \mathrm{MHz}$ center frequency and 12 cycles in a Hanning window using Labview and an arbitrary function generator. The signal was amplified using a power amplifier and applied to the piezoelectric transducer. Measurements were performed using a non-contact laser interferometer. A band pass filter from 2 to $7 \mathrm{MHz}$ was applied and the averaged signal (50 averages) was recorded using a digital storage oscilloscope for each measurement point. Phase velocity measurements were performed in the far field at a distance of $42 \mathrm{~mm}$ from the angle beam transducer over a straight line of $10 \mathrm{~mm}$ with measurement steps every $0.2 \mathrm{~mm}$, in total 51 measurement points. This was repeated from the $<100>$ crystal orientation in $5^{\circ}$ angular steps over $45^{\circ}$ to the $<110>$ crystal direction.

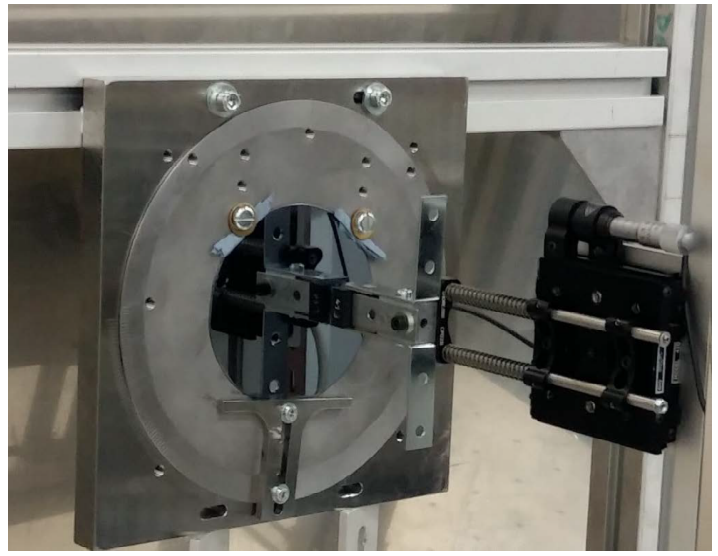

Fig. 2: Experimental setup with silicon wafer on holder and transducer holder. 

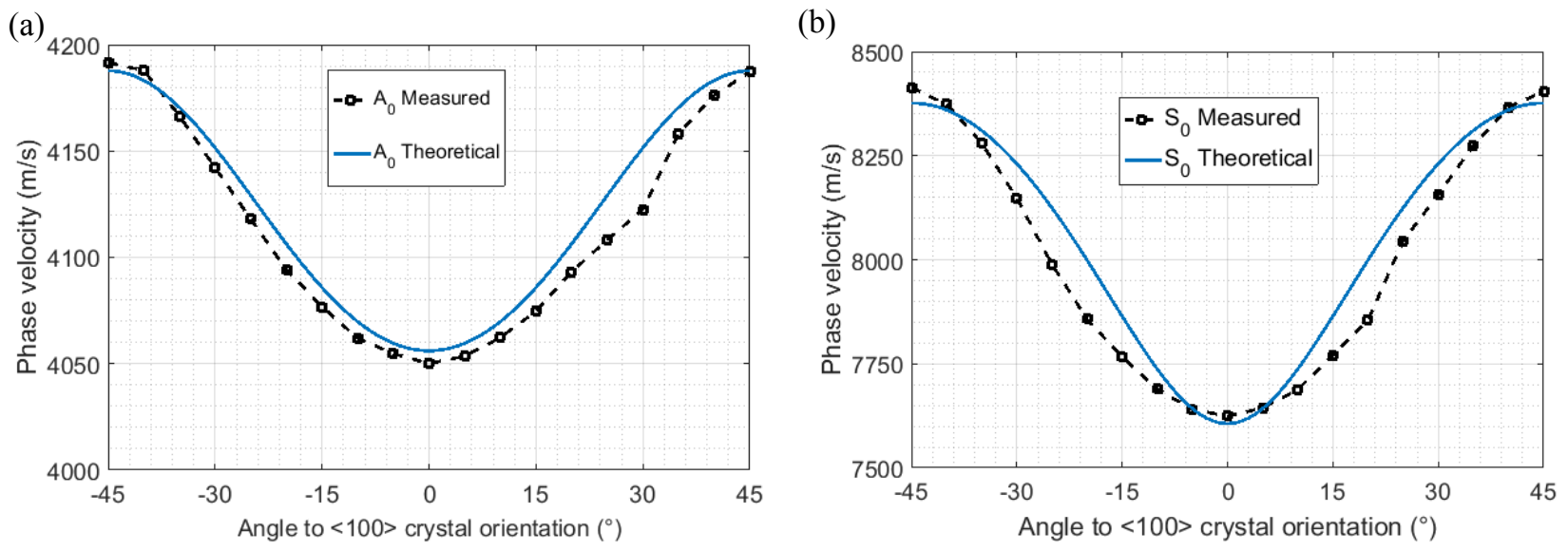

Fig. 3: Variation of phase velocity with propagation direction, theory (Disperse) and measured; (a) $\mathrm{A}_{0}$ mode; (b) $\mathrm{S}_{0}$ mode.

The phase velocity $\left(\mathrm{c}_{\mathrm{p}}\right)$ was evaluated by applying Fast Fourier Transform (FFT) to the time gated signal at every measurement point. The phase values at the excitation frequency were extracted. After removal of phase jumps, the phase variation in function of the spatial measurement step allows the calculation of the phase velocity. Beam skew measurements were performed using the same setup with the laser measurement over a 2D area, 21 steps of $1 \mathrm{~mm}$ perpendicular to the beam propagation and 9 steps of $5 \mathrm{~mm}$ along wave propagation direction.

\section{PHASE SLOWNESS}

The theoretical phase velocity was predicted using Disperse for the nominal orthotropic material properties of the silicon wafers and is shown in Fig. 3. For the $\mathrm{A}_{0}$ mode a 3\% variation was predicted and the measured values match the theoretical values very well and show the expected symmetry to the $0^{\circ}$ orientation, with a small measurement error in the $30^{\circ}$ direction. For the $\mathrm{S}_{0}$ mode a larger variation of the phase velocity with orientation is predicted theoretically and matched from the measurement results in the $0^{\circ}$ and $45^{\circ}$ directions. However, a small, systematic offset was found in the non-crystallographic directions, with lower phase velocity values than theoretically predicted, probably linked to the significant wave skew of the $\mathrm{S}_{0}$ mode for these directions.

\section{WAVE SKEW}

The displacement on a rectangular grid was recorded to quantify the excited wave beam. The amplitude was evaluated at each point of the grid by taking the maximum of the Hilbert transform for the experimental results and amplitude at 5 $\mathrm{MHz}$ center frequency (FFT) for the simulation results. These are shown in Fig. 4 for the FE simulations and in Fig. 5 for the measurements of the $A_{0}$ and $S_{0}$ modes. For the $A_{0}$ mode a strong beam can be observed for all crystallographic orientations, with a small wave skew for the $15^{\circ}$ and $30^{\circ}$ directions and a slightly larger beam widening for the $0^{\circ}$ direction. The wave skew for the $\mathrm{S}_{0}$ mode for the $15^{\circ}$ and $30^{\circ}$ directions is significantly higher. Beam widening for the $\mathrm{S}_{0}$ mode can be clearly seen, with a larger amplitude decrease for the $<100>$ orientation than for the $<110>$ direction. Overall a good match can be seen for the FE simulations and experiments with the same overall effects. The match of the beam width and spreading is quite similar, meaning that the wedge excitation was modelled with sufficient accuracy using the line excitation in the FE analysis. The wave skew and beam spread were evaluated for each orientation. As expected from the phase slowness curves (Fig. 1b) the predicted maximum wave skew for the $\mathrm{S}_{0}$ mode with 10.6 degrees is significantly larger than for the $\mathrm{A}_{0}$ mode with 3.6 degrees. As the wave propagation for the FE simulations is perfectly symmetric for the $0^{\circ}$ and $45^{\circ}$ directions, no wave skew was observed. For the experimental results the measurement results for the $<110>$ and $<100>$ crystallographic orientations are not perfectly symmetric and a slight skew was seen in Fig. 5. This was found to be up to $0.3^{\circ}$ for the $A_{0}$ mode and $1.9^{\circ}$ for the $S_{0}$ mode. This is most likely due to a small misalignment of the wedge transducer to the crystal orientation, which could only be achieved with about 1 degree accuracy. 

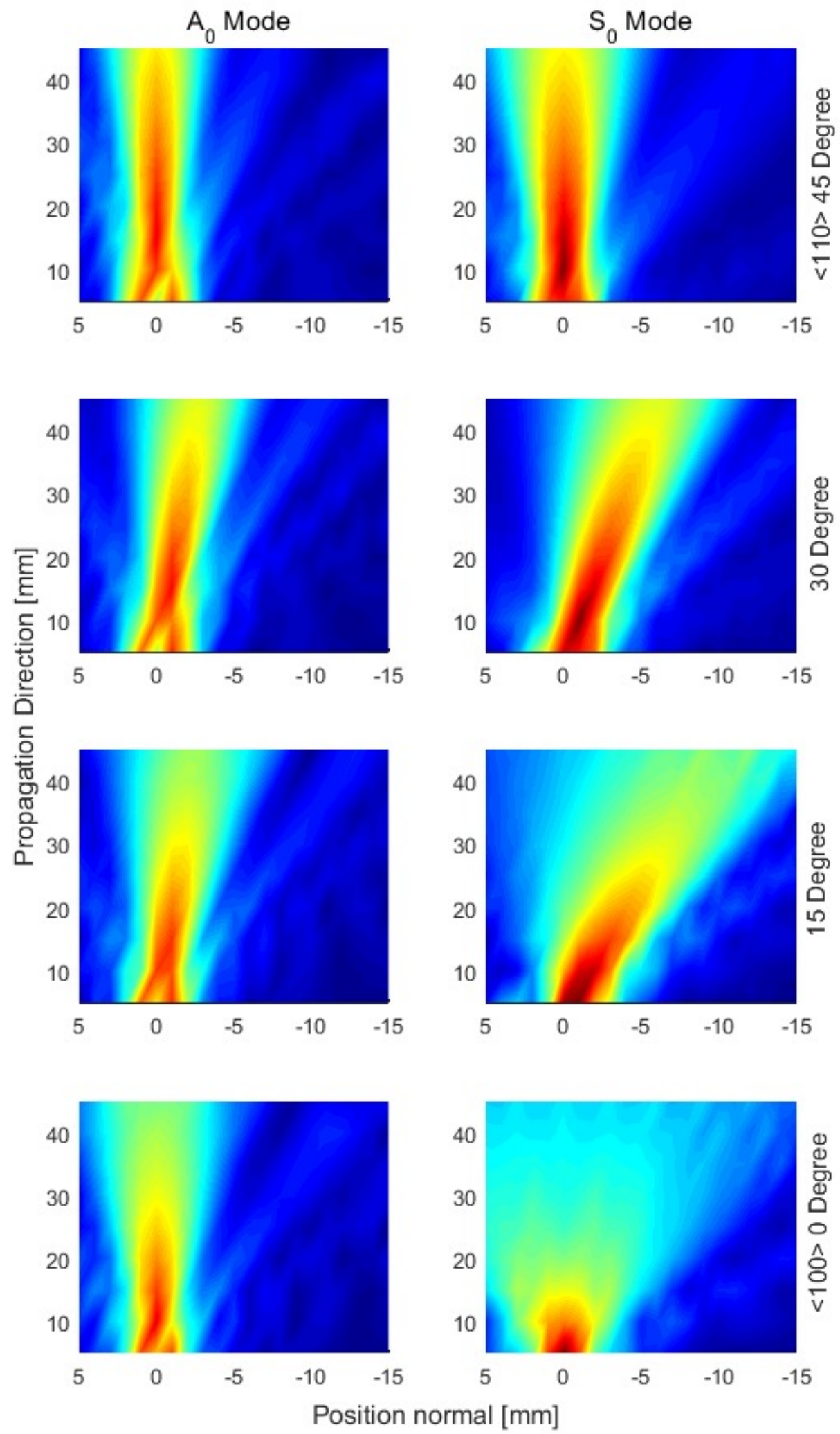

Fig. 4: FE simulation of guided wave beam skewing; evaluation of amplitude at $5 \mathrm{MHz}$ center frequency (FFT) at each monitoring location for $\mathrm{A}_{0}$ and $\mathrm{S}_{0}$ mode, different crystallographic orientations relative to line excitation. 


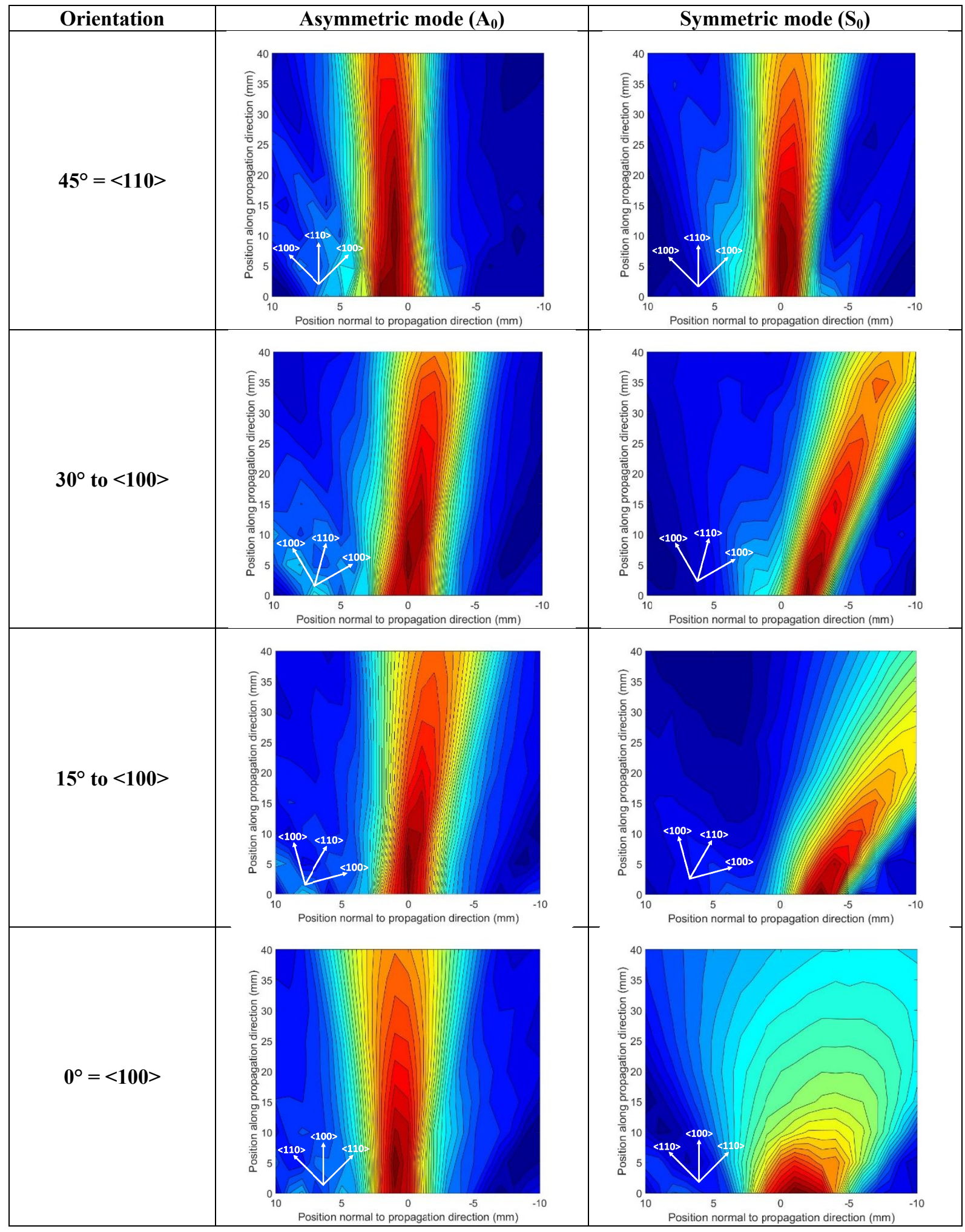

Fig. 5: Measurement of guided wave beam skewing; evaluation of amplitude (maxima of Hilbert transform) at each monitoring location for $\mathrm{A}_{0}$ and $\mathrm{S}_{0}$ mode, different crystallographic orientations relative to transducer orientation. 


\section{CONCLUSIONS}

Guided wave propagation in monocrystalline silicon wafers was simulated using 3D FE models and measured experimentally. The material anisotropy leads to variations of the wave characteristics, depending on the propagation direction relative to the crystal orientation. The measured phase velocity (slowness) and skew angle of the two fundamental Lamb wave modes (first antisymmetric mode $\mathrm{A}_{0}$ and first symmetric mode $\mathrm{S}_{0}$ ) for varying propagation directions relative to the crystal orientation were analyzed and compared to predictions. Good agreement based on nominal material properties of the silicon wafer was found. The phase velocity of the first antisymmetric Lamb wave mode $\left(\mathrm{A}_{0}\right)$ varies by a small amount depending on the propagation direction to the crystal orientation and minor skewing of the guided wave beam was observed. Significantly larger variations of the phase velocity of the $\mathrm{S}_{0}$ mode with propagation direction were predicted and found experimentally, leading to a significant wave skew phenomenon and widening of the guided wave beam. This indicates that the variation of the guided wave propagation due to the material anisotropy has to be considered for the non-destructive monitoring of silicon wafers.

\section{REFERENCES}

[1] Luque, A. and Hegedus, S., [Handbook of Photovoltaic Science and Engineering], Wiley, New York (2011).

[2] Abdelhamid, M., Singh, R. and Omar, M., "Review of microcrack detection techniques for silicon solar cells," IEEE J. Photovoltaics 4, 514-524 (2014).

[3] Israil, M., Ghani, A. and Kerm, Y., "Non-destructive microcracks detection techniques in silicon solar cell," Phys. Sci. Int. J. 4, 1073-1087 (2014).

[4] Rose, J.L., "Standing on the shoulders of giants: An example of guided wave inspection," Mat. Eval. 60, 53-59 (2002).

[5] Fromme, P., "Monitoring of Plate Structures Using Guided Ultrasonic Waves," AIP Conf. Proc. 975, 78-85 (2008).

[6] Fromme, P., "Health Monitoring of Plate Structures using Guided Waves," Proc. SPIE 6935, 69350W (2008).

[7] Masserey, B., Raemy, C. and Fromme, P., "High-frequency guided ultrasonic waves for hidden defect detection in multi-layered aircraft structures," Ultrasonics 54, 1720-1728 (2014).

[8] Chan, H., Masserey B. and Fromme, P., "High frequency guided ultrasonic waves for hidden fatigue crack growth monitoring in multi-layer model aerospace structures," Smart Mater. Struct. 24, 025037 (2015).

[9] Masserey, B. and Fromme, P., "Fatigue Crack Growth Monitoring using High Frequency Guided Waves," Struct. Health Monit. 12, 484-493 (2013).

[10] Masserey, B. and Fromme, P., "In-Situ Monitoring of Fatigue Crack Growth using High Frequency Guided Waves," NDT\&E Int. 71, 1-7 (2015).

[11] Song, M.-K. and Jhang, K.-Y., "Crack Detection in Single-Crystalline Silicon Wafer Using Laser Generated Lamb Wave," Adv. Mater. Sci. Eng. 2013, 950791 (2013).

[12] Chakrapani, S.K., Padiyar, M.J. and Balasubramaniam, K., "Crack detection in full size Cz-silicon wafers using lamb wave air coupled ultrasonic testing (LAC-UT)," J. Nondestruct. Eval. 31, 46-55 (2012).

[13] Maris, H.J., "Enhancement of heat pulses in crystals due to elastic anisotropy," J. Acoust. Soc. Am. 50, 812-818 (1971).

[14] Kim, K.Y., Bretz, K.C., Every, A.G. and Sachse, W. "Ultrasonic imaging of the group velocity surface about the cubic axis in silicon,” J. Appl. Phys. 79, 1857-1863 (1996).

[15] Audoin, B., Bescond, C. and Deschamps, M., "Measurement of stiffness coefficients of anisotropic materials from pointlike generation and detection of acoustic waves," J. Appl. Phys. 80, 3760-3771 (1996).

[16] Prada, C., Clorennec, D., Murray, T.W. and Royer, D., "Influence of the anisotropy on zero-group velocity Lamb modes," J. Acoust. Soc. Am. 126, 620-625 (2009).

[17] Veidt, M. and Sachse, W., "Ultrasonic point-source/point-receiver measurements in thin specimens," J. Acoust. Soc. Am. 96, 2318-2326 (1994).

[18] Leleux, A., Micheau, P. and Castaings, M., "Long range detection of defects in composite plates using Lamb waves generated and detected by ultrasonic phased array probes," J. Nondestruct. Eval. 32, 200-214 (2013).

[19] Chapuis, B., Terrien, N. and Royer, D. "Excitation and focusing of Lamb waves in a multilayered anisotropic plate," J. Acoust. Soc. Am. 127, 198-203 (2010).

[20] Pavlakovic, B., Lowe, M.J.S., Alleyne, D. and Cawley, P., "Disperse: A general purpose program for creating dispersion curves," Proc. QNDE 16, 185-192 (1997).

[21] Rose, J.L., [Ultrasonic waves in solid media], Cambridge University press, Cambridge (1999). 